\title{
The Cytomegalovirus Enhancer Induces an Immediate Response to the Myosin Light Chain 2v Promoter during P19CL6 Cell Differentiation
}

\author{
Takanari Wakayama1, Kazuaki Ohashi², Yasuyuki Fujimoto3 ${ }^{3}$, Masatomo Maeda ${ }^{*}$ \\ ${ }^{1}$ Laboratory of Biochemistry and Molecular Biology, Graduate School of Pharmaceutical Sciences, Osaka University, Osaka, Japan \\ ${ }^{2}$ Department of Medical Biochemistry, School of Pharmacy, Iwate Medical University, Shiwa-Gun, Japan \\ ${ }^{3}$ Department of Molecular Biology, School of Pharmacy, Iwate Medical University, Shiwa-Gun, Japan \\ ${ }^{4}$ Laboratory of Gene Therapy, Faculty of Pharmaceutical Sciences, Niigata University of Pharmacy and Applied Life Sciences, \\ Niigata, Japan \\ Email:mmaeda@nupals.ac.jp
}

How to cite this paper: Wakayama, T., Ohashi, K., Fujimoto, Y. and Maeda, M. (2017) The Cytomegalovirus Enhancer Induces an Immediate Response to the Myosin Light Chain 2v Promoter during P19CL6 Cell Differentiation. American Journal of Molecular Biology, 7, 190-203. https://doi.org/10.4236/ajmb.2017.74015

Received: August 22, 2017

Accepted: October 17, 2017

Published: October 20, 2017

Copyright $\odot 2017$ by authors and Scientific Research Publishing Inc. This work is licensed under the Creative Commons Attribution International License (CC BY 4.0).

http://creativecommons.org/licenses/by/4.0/

c) (i) Open Access

\begin{abstract}
The P19CL6 mouse embryonic carcinoma cells efficiently differentiate into cardiac muscle cells in the presence of DMSO. A reporter plasmid for cardiac muscle differentiation was constructed by connecting the CMV enhancer and a 250 bp MLC-2v promoter in front of the GFP gene to further evaluate the role of the CMV enhancer. This plasmid (pCBVenh/MLC-2 $\mathrm{v}_{\mathrm{pro}} / \mathrm{EGFP}$ ) was stably introduced into P19CL6 cells, and the transfectant differentiated into cardiomyocytes with DMSO. Upon DMSO addition, GFP was immediately transcribed (within 2 days) and the amount of the transcript increased with cultivation. Concomitantly, GFP fluorescence was detected in the cells under a microscope. However, native MLC-2v was transcribed later on day 4. This expression time course is different from that of GFP. Clearly the CMV enhancer responded immediately to DMSO. Since GATA DNA-binding proteins play crucial roles in the initiation of cardiomyocyte differentiation, such a response could be ascribed to the presence of multiple GATA motifs in the enhancer sequence but not in the native MLC-2v promoter. Thus the CMV enhancer may be not only useful for gene therapy and monitoring cell differentiation but also the study of the role of GATA transcription factors expressed in P19CL6 cells.
\end{abstract}

\section{Keywords}

Cytomegalovirus Enhancer, Differentiation, GATA Transcription Factor, 
Gene Expression, Heart Muscle, MLC-2v, P19CL6 Cells, Promoter

\section{Introduction}

P19CL6 cells derived from P19 embryonic carcinoma cells can efficiently differentiate into cardiac muscle cells in the presence of $1 \%$ dimethyl sulfoxide (DMSO) [1]. Such a property is a good tool for the study of cardiac myocyte differentiation in vitro. Before transcriptional activation of the genes for cardiac contractile proteins during the differentiation of P19CL6 cells, the gene for the GATA-4 transcription factor becomes activated [2]. We have also been reported that the GATA- 4 gene is immediately transcribed upon addition of DMSO through binding of GATA- 6 to the upstream enhancer GATA motif, which is conserved in mammals [3]. Not only the GATA-4 but also the MEF2C and Tbx5 transcription factors play crucial roles in cardiac myocyte differentiation [4]. Furthermore, genetic analysis suggested that NKX2.5 and GATA- 6 together with these three transcription factors are essential for cardiac development [5] [6].

As for contractile proteins, transcripts of myosin heavy chains ( $\alpha$-MHC and $\beta$-MHC) were detected in parallel in P19CL6 cells at a late stage after the start of cardiac differentiation [1]. Similar to these cardiac MHC isoforms, cardiac myosin light chain 2 (MLC-2v) is also transcribed during the course of differentiation [7]. When the 250 base pair (bp) promoter for the rat cardiac MLC-2v gene was connected upstream of the gene for green fluorescent protein (GFP) together with the enhancer portion of the cytomegalovirus (CMV) immediate early promoter, and the resulting reporter plasmid was stably introduced into P19CL6 cells, expression of GFP was limited to developing cardiac myocytes [8]. Although this system could be advantageous to quantify cardiac differentiation, it has not been addressed as to why the virus enhancer responds to such a differentiation signal. Actually, it has been demonstrated that the SV40 enhancer acted on both heart muscle and non-muscle cells [9].

In this study, we analyzed the MLC- $2 \mathrm{v}$ promoter driven by the CMV enhancer in more details. Although the enhancer-promoter construct responded to the DMSO signal, the response was immediately before the start of transcription of native MLC-2v. We will discuss the role(s) of GATA motifs in the CMV enhancer sequence from the viewpoint of GATA-4 transcription in P19CL6 cells [3].

\section{Materials and Methods}

\subsection{Construction of an Expression Plasmid for GFP under the Control of the MLC-2v Promoter.}

pEGFP-N1 (Clonetech) was EcoRI-digested, and then its CMV enhancer moiety (nucleotide residue numbers 60 - 465, GenBank Accession No. U55762) was amplified by means of polymerase chain reaction (PCR) [10] with a primer pair, 
CMVenh-F/CMVenh-R, and Pyrobest DNA polymerase (TaKaRa) [preheating $\left(94^{\circ} \mathrm{C}, 5 \mathrm{~min}\right)$, followed by 30 cycles of denaturation $\left(94^{\circ} \mathrm{C}, 15 \mathrm{sec}\right)$, annealing $\left(55^{\circ} \mathrm{C}, 30 \mathrm{sec}\right)$, and extension $\left(72^{\circ} \mathrm{C}, 30 \mathrm{sec}\right)$, and then post-heating $\left(72^{\circ} \mathrm{C}, 5\right.$ min)]. The product was cloned into the SmaI site of pBluescript II SK(+) (Stratagene). To amplify the MLC-2v promoter [11], rat liver genomic DNA [12] was digested with EcoRI and then subjected to PCR with a primer pair, MLC-2v $\mathrm{p}_{\text {pro }}{ }^{-}$ F/MLC- $2 \mathrm{v}_{\text {pro }}-\mathrm{R}$, and LA Taq DNA polymerase (TaKaRa) [preheating $\left(94^{\circ} \mathrm{C}, 5\right.$ $\mathrm{min})$, followed by 30 cycles of denaturation $\left(94^{\circ} \mathrm{C}, 15 \mathrm{sec}\right)$, annealing $\left(52^{\circ} \mathrm{C}, 30\right.$ sec), and extension $\left(72^{\circ} \mathrm{C}, 30 \mathrm{sec}\right)$, and then post-heating $\left.\left(72^{\circ} \mathrm{C}, 5 \mathrm{~min}\right)\right]$. The product was treated with T4 DNA polymerase (Toyobo), and then cloned into the SmaI site of pBluescript II SK(+). The $406 \mathrm{bp}$ Asel-HindIII fragment of the CMV enhancer and the $262 \mathrm{bp}$ HindIII-BamHI fragment of the MLC-2v promoter were inserted into a large fragment of pEGFP-N1 (4079 bp) digested with AseI and BamHI. The resulting plasmid was named pCMVenh/MLC-2 $\mathrm{v}_{\mathrm{pro}} / \mathrm{GFP}$ (4759 bp), and confirmed by the production of a 406 bp HindIII-Asel fragment, and a $1008 \mathrm{bp} \mathrm{NotI}$ and HindIII fragment (Figure 1). The entire nucleotide sequence of pCMVenh/MLC- $2 \mathrm{v}_{\mathrm{pro}} / \mathrm{GFP}$ was shown in Figure S1 (Supplementary).

The cloned DNA was sequenced by the dideoxy chain-termination method [13] with a primer (M13 forward or M13 reverse) and a BigDye Terminator v3.1 Cycle Sequencing Kit (Applied Biosystems). The oligonucleotides used for PCR and sequencing are listed in Table 1 . The molecular biological techniques were performed by published methods [14].

\subsection{Cell Culture}

P19CL6 cells [3] were cultured in $\alpha$-Eagle's minimal essential medium (GIBCO

Table 1. Sequences of oligonucleotides used in this study.

\begin{tabular}{|c|c|}
\hline & Asel \\
\hline \multirow[t]{2}{*}{ CMVenh-F } & 5'-TTA TTA ATG CGT TAC ATA ACT TAC GGT AAA-3' \\
\hline & HindIIII \\
\hline \multirow[t]{2}{*}{ CMVenh-R } & 5'-TTA AGC TTC AAA ACA AAC TCC CAT TGA CG-3' \\
\hline & HindIII \\
\hline \multirow[t]{2}{*}{$M L C-2 v_{\text {pro }}-F$} & 5'-TTA AGC TTG ACC CAG AGC ACA GAG CAT-3' \\
\hline & $\mathrm{BamHI}$ \\
\hline$M L C-2 v_{p r o}-R$ & 5'-TTG GAT CCAAGG AGC CTG CTG GCC GG-3' \\
\hline MLC-2v-S & 5'-GCC AAG AAG CGG ATA GAA GG-3' \\
\hline MLC-2v-A & 5'-CTG TGG TTC AGG GCT CAG TC-3' \\
\hline GFP-F & 5'-TGC AGT GCT TCA GCC GCT A-3' \\
\hline GFP-R & 5'-TTG TAC AGC TCG TCC ATG CC-3' \\
\hline YSactin-S & 5'-GCA GGA GAT GGC CAC TGC CGC-3' \\
\hline YSactin-A & 5'-TCT CCT TCT GCA TCC TGT CAG C-3' \\
\hline \multicolumn{2}{|c|}{ Sequence Primer } \\
\hline M13-F & 5'-CGC CAG GGT TTT CCC AGT CAC GAC-3' \\
\hline M13-R & 5'-GAG CGG ATA ACA ATT TCA CAC AGG-3' \\
\hline
\end{tabular}

Restriction enzyme sites are underlined with bold letters. 

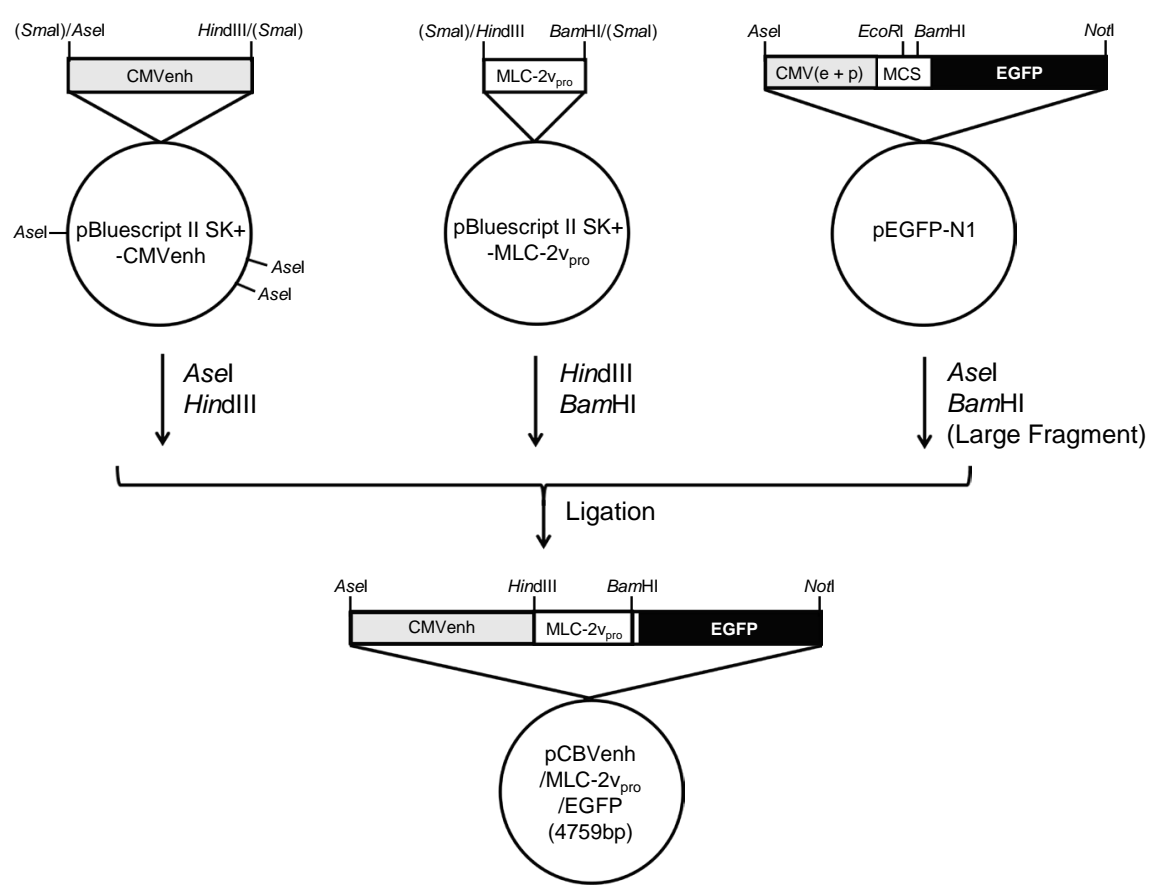

Figure 1. Construction of an expression plasmid for GFP with the CMV enhancer and MLC-2v promoter. The CMV enhancer moiety (CMVenh, $406 \mathrm{bp}$ ) derived from pEGFP$\mathrm{N} 1$ and the MLC-2v promoter (MLC-2 $\mathrm{v}_{\text {pro }}, 262 \mathrm{bp}$ ) cloned from rat liver genomic DNA were inserted into the pEGFP-N1 vector from which the human cytomegalovirus (CMV) immediate early enhancer and promoter sequences $[\mathrm{CMV}(\mathrm{e}+\mathrm{p})]$ corresponding to nucleotide residue numbers 1 - 589 (GenBank Accession No. U55762) had been deleted. Typical restriction enzyme sites used in this study (AseI, BamHI, EcoRI, HindIII, NotI and SmaI) were indicated in the figure. MCS, multi-cloning site.

BRL) supplemented with $10 \%(\mathrm{v} / \mathrm{v})$ fetal bovine serum (GIBCO BRL) and antibiotics $(100 \mu \mathrm{g} / \mathrm{mL}$ streptomycin sulfate and $100 \mathrm{u} / \mathrm{mL}$ benzyl-penicillin) (Wako). An expression plasmid was introduced into the cells $\left(10^{5}\right.$ cells per $6 \mathrm{~cm}$ diameter dish) by means of the calcium-phosphate method as described previously [15]. Cells were split into $10 \mathrm{~cm}$ diameter dishes after $48 \mathrm{hrs}$ incubation, and then grown in the presence of $1 \mathrm{mg} / \mathrm{mL}$ G418 (Nacalai). Among six resistant colonies, one (clone B4) that showed strong green fluorescence in the presence of $1 \%(\mathrm{v} / \mathrm{v})$ DMSO was analyzed.

The B4 clone $\left(10^{4}\right.$ cells) was seeded into a $6 \mathrm{~cm}$ diameter dish. Cells were grown in the medium plus $1 \%(\mathrm{v} / \mathrm{v})$ DMSO. The medium was changed to fresh medium containing DMSO on the fourth day, and then every two days. The expression of GFP was monitored under a microscope (Olympus IX-70) equipped with an AQUACOSMOS U7501 (HAMAMATSU PHOTONICS).

\subsection{Determination of the Expression Levels of mRNAs}

Cells $\left(10^{4}\right.$ cells) were seeded into a $6 \mathrm{~cm}$ diameter dish. Total RNA was extracted with Isogen (Nippon Gene), and an aliquot ( $5 \mu \mathrm{g}$ ) was reverse transcribed with M-MLV reverse transcriptase (TaKaRa) and an oligo $(\mathrm{dT})_{15}$ primer. After RNaseH (TaKaRa) treatment, cDNA was subjected to semi-quantitative PCR with 
Go $\operatorname{Taq}^{\circledR}$ (Promega) and a primer pair, MLC-2v-S/MLC-2v-A, GFP-F/GFP-R or YSactin-S/YSactin-A (Table 1): the PCR conditions comprised preheating $\left(94^{\circ} \mathrm{C}, 3 \mathrm{~min}\right)$, followed by denaturation $\left(94^{\circ} \mathrm{C}, 0.5 \mathrm{~min}\right)$, annealing $\left[\left(60^{\circ} \mathrm{C}\right.\right.$ for MLC-2v and GFP, and $55^{\circ} \mathrm{C}$ for $\beta$-actin), $0.5 \mathrm{~min}$ ] and extension $\left(72^{\circ} \mathrm{C}, 0.5 \mathrm{~min}\right)$, and then post-incubation $\left(72^{\circ} \mathrm{C}, 5 \mathrm{~min}\right)$. The PCR products were size-separated on an agarose gel and DNA bands were visualized with ethidium bromide. Images were recorded with a FAS-III UV-imaging system (Toyobo).

\subsection{Chemicals}

Restriction enzymes were obtained from NEB and Toyobo. Agarose LO3 and a DNA ligation kit ver. 2.0 were purchased from TaKaRa. A GENECLEAN ${ }^{\circledR}$ III KIT and oligonucleotides were provided by MP Biomedicals and Gene Design Inc., respectively. All other chemicals used were of the highest grade commercially available.

\section{Results}

\subsection{Expression of GFP in the Stable Transfectant Cells}

We constructed an expression plasmid for GFP under the control of the CMV enhancer and MLC-2v promoter (Figure 1). This expression plasmid was stably introduced into P19CL6 cells and a G418-resistant clone carrying the reporter gene was isolated. The clone was cultured in the presence and absence of $1 \%$ DMSO, and green fluorescence derived from GFP was monitored under a microscope. As shown in Figure 2, weak fluorescence was detected on day 4 in the presence of DMSO. The fluorescence increased gradually and was much stronger on day 12 under the differentiation conditions. However, without DMSO only a background level of fluorescence was detected on day 12. Previous study also showed that very little but detectable amounts of GFP was expressed in undifferentiated cells [8].

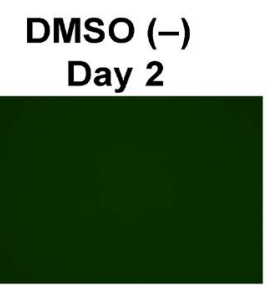

Day 12

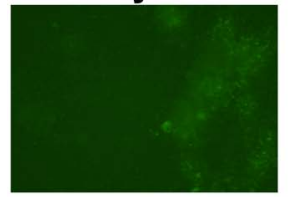

DMSO (+)

Day 2

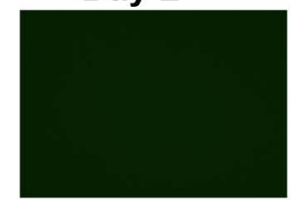

Day 8

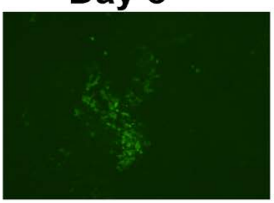

Day 4

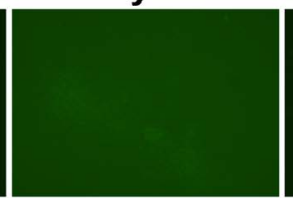

Day 10

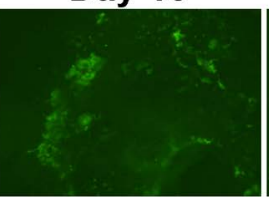

Day 6

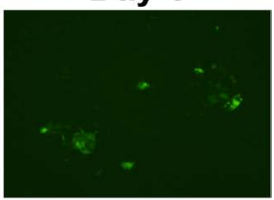

Day 12

Figure 2. Expression of GFP in the stable transfectant clone derived from P19CL6 cells. The expression plasmid (pCBVenh/MLC- $2 \mathrm{v}_{\mathrm{pro}} / \mathrm{EGFP}$ ) constructed as in Figure 1 was stably introduced into P19CL6 cells. The G-418 resistant clone was cultured in the presence [DMSO (+)] or absence [DMSO (-)] of $1 \%(\mathrm{v} / \mathrm{v})$ DMSO. Fluorescence images on the indicated days after the start of the experiment are shown. Bar, $200 \mu \mathrm{m}$.

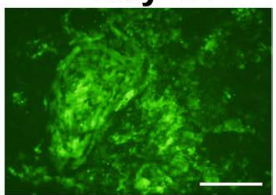


To determine the start of transcription of the GFP gene, total RNA was extracted and subjected to semi-quantitative RT-PCR. The results of the cDNA amplification indicated that the gene for GFP had already been transcribed on day 2 after the addition of DMSO, whereas it was not detected in the absence of DMSO (compare the results on days $2(+)$ and $4(+)$ with those of day $4(-)$, Figure 3, upper panel). Furthermore, the amount of the transcript of GFP clearly increased up to day $12(+)$. Although we did not determine the GFP protein by means of Western blotting, the transcript of GFP was translated as shown in Figure 2.

\subsection{Comparison of the Expression of Native MLC-2v and GFP}

The expression pattern of GFP was compared with that of native MLC-2v. As shown in Figure 3 (middle panel), the start of transcription of the native MLC-2v was clearly retarded in contrast to the appearance of the transcript of GFP. The transcript of MLC-2v appeared abruptly on day 4, and its amount was maintained constantly. Since the rat and mouse MLC-2v prompter sequences are essentially the same (Figure 4(a)), it is suggested that the immediate transcription of the GFP reporter gene could not be ascribed to the MLC-2v prompter moiety but rather to the CMV enhancer.

\subsection{Presence of GATA Motifs in the CMV Enhancer}

Since the CMV enhancer started to operate immediately after DMSO addition (Figure 3), it seems likely that the potential master regulator(s) that directs P19CL6 cells toward cardiomyocytes may instantaneously bind to and activate the enhancer in the presence of DMSO. Our previous study demonstrated that the binding of pre-existing GATA- 6 to an upstream GATA motif is essential for the immediate activation of the GATA-4 gene upon DMSO addition to P19CL6

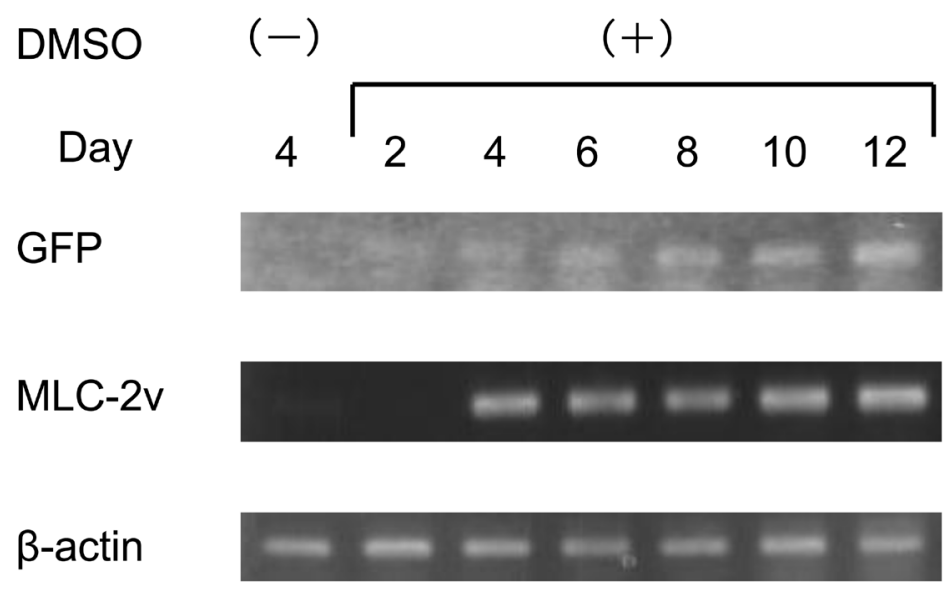

Figure 3. Detection of transcripts of GFP and native MLC-2v. Total RNA was prepared on the indicated days after DMSO addition, and then subjected to RT-PCR to detect transcripts of GFP and native MLC-2v. $\beta$-actin mRNA was used as a positive control. Amplified fragments were analyzed by $2 \%(\mathrm{w} / \mathrm{v})$ agarose gel-electrophoresis. 
(a)

\begin{tabular}{|c|c|c|c|}
\hline & & & \\
\hline Iuman & -225 & cgcagagagcgcgcagcatctctccccgtgaccatga-cccagctactgcctctttaaccttgaatgcctttttggggg & -147 \\
\hline lat & -251 & gacccagagcacagagcatc-gttcccagg-ccaggc-cccagccactgtctctttaaccttgaaggcatttttgggt- & -176 \\
\hline lou & -259 & ggaagagagcacaccccatca-t-ccccaggccaggc-cccagccactgactctttaaccttgaaggcatttttgggt- & -184 \\
\hline & -238 & ctgcccactcga-gtgtagctcgtgggagctgcagggcctcctggtgtg-ct-gctaaccttgaaagcctctgt--gt- & -282 \\
\hline & & E-box $* \quad * * * * * \underset{\mathrm{HF}-2}{* * *} * * * * * * * * * * * * * * *$ & \\
\hline uman & -146 & 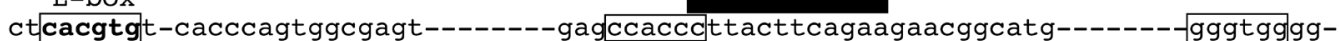 & -85 \\
\hline at & -175 & ctcacgtgt 4 ccaccdaggcgggtgtcggactttgaacggctcttacttcagaagaacggcatg-- & -105 \\
\hline ous & -183 & tcacgtgtccaccdaggcgggtggccgcctttgagcggetcttact & -107 \\
\hline & -164 & ctcgcatgt-cacgtcggccagtgac-aac----agcaag-cttactgcagattagaggaggagac & -92 \\
\hline & & 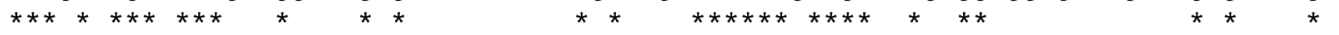 & \\
\hline & & $\mathrm{HF}-1$ & \\
\hline uman & -84 & 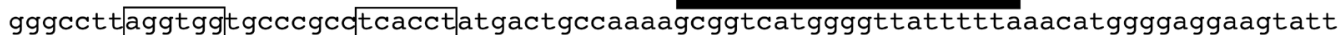 & -5 \\
\hline lat & -104 & gggc-ttlaggtggcctctgcdtcacctacaactgccaaaagtggtca & -26 \\
\hline ou, & -106 & gggc-ttlaggtggectccgcdtcacctacaactgccaaaagtggtca & -28 \\
\hline Chicken & -91 & ggg---tcgggggtggctggtttacctattacagccaaaagtggacatggggttatttttagcctggaatggggtgtatt & -15 \\
\hline & & $* * * \quad * * * * * \quad * * * * * * * * \quad * * \quad * * * * * * * * * * * \quad * * * * * * * * * * * * * * * * \quad *$ & \\
\hline & -4 & ttcctgggctgcagag--agctgggcggagtgtggaat & +74 \\
\hline & -25 & attgttccacagcaggggcc--ggccagcaggctccttgaattctt & +50 \\
\hline & -27 & ttccacagcaggggcagaggccágcaggctcct & +50 \\
\hline cken & -14 & =tgtttcagcccagggaggcagagggactgcctctgagatccctcctgcagcaccaccagcac & +66 \\
\hline & & 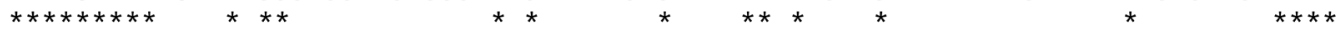 & \\
\hline
\end{tabular}

(b)

Figure 4. GATA motifs in the CMV enhancer, and comparison of vertebrate MLC-2v promoter sequences. (a) The CMV enhancer sequence (nucleotide residues 60 - 465) in pEGFP-N1 (GenBank Accession No. U55762) is shown. The positions of GAT(A/T) motifs are boxed. Similar sequences of the canonical NKX2.5 and Tbx5 binding sites [23] [24] (5'-TYAAGTG-3' and 5'-(A/G) GGTGT-3', respectively) are double- and single-underlined, respectively; (b) Upstream sequences of the genes for human, rat and mouse MLC-2v [NCBI Reference Sequences NG_007554 and NM_001035252, and GenBank Accession No. AF326769, respectively], and chicken $\mathrm{MLC}_{2 \mathrm{~A}}$ [29] were aligned. The transcribed sequences are shown in italics. Initiation codons are indicated by capital letters. Vertical arrowheads indicate the transcriptional start sites. Residues conserved in all four vertebrates are denoted by asterisks. Two arrows indicate the sequence primers for amplifying the rat promoter sequence (262 bp) between nucleotide residues -250 and +12 . Thick overlines labeled HF-1-HF-3 are conserved regions that were characterized previously [27]. A typical E-box sequence (CANNTG) [27] and closely related Tbx5 binding sites [5'-(A/G)GGTGT-3'] [24] are boxed.

cells [3]. Consistent with this observation, the CMV enhancer has multiple GATA-6 binding motifs, 5'-GAT(A/T)-3' [16]; two GATA and one GATT sequence are present in the downstream half of the $406 \mathrm{bp}$ enhancer sequence (Figure 4(a)). However, such a motif could not be found in the $262 \mathrm{bp} \mathrm{MLC-2v}$ promoter sequence (Figure 4(b)). Since GATA-6 could activate reporter genes through GAT(A/T) sequences [17] [18], GATA-6 may bind to the CMV enhance in response to a cardiac differentiation signal. 


\section{Discussion}

P19CL6 cells are used as a model of differentiation of cardiac myoblasts into myocytes [1] [8]. In this study, we found that the MLC-2v promoter harboring the CMV enhancer responded immediately in P19CL6 cells upon the addition of DMSO, which is an induction reagent for cardiac differentiation [1]. However, the intrinsic MLC-2v promoter was activated rather later during the differentiation (Figure 3). Thus, the transcription of GFP under the control of the CMV enhancer is not a result of cardiac myocyte differentiation, but rather parallel to activation of the start of differentiation. It should be noted that such comparison between native and hybrid MLC-2v promoters focused on the CMV enhancer has not been carried out previously [8].

As for GATA-4 gene expression, binding of GATA-6 to the distal enhancer GATA motif was obligatory for transcriptional activation under the differentiation conditions for P19CL6 cells [3]. Since GATA-4 and GATA-6 function in transcription through a GAT(A/T) motif [17] [18], it seems likely that the CMV enhancer with GATA motifs (Figure 4(b)) immediately responds to pre-existing GATA-6 in P19CL6 cells on DMSO treatment. Actually, the CMV enhancer induces efficient cell-type specific expression of genes such as those of atrial natriuretic factor and MLC-2v in cardiomyocyte cell line HL-1 [19], which expresses GATA-4 and GATA-6 [20]. Consistent with this finding, the SV40 enhancer composed of the $72 \mathrm{bp}$ tandem repeat [21] without GATA motifs (NCBI Reference Sequence NC_001669) abolished the cardiac specificity [9].

The gradual increases in the amounts of GFP transcripts shown in Figure 3 could be explained by the participation of the pre-existing GATA-6, and increased amounts of GATA-4 and GATA-5 arising through transcription and translation [3]. It is also suggested that factors like GATA-4 that potentiate transcription during development are inherently capable of initiating chromatin opening through binding to an enhancer [22]. Thus, the CMV enhancer with GATA motifs may become open and activated in an environment in which GATA factors are expressed although modulation and/or a trigger such as DNA methylation and protein modification could be needed by DMSO.

In the CMV enhancer (Figure 4(a)), there are sequence motifs similar to the canonical binding sites for NKX2.5 and Tbx5, 5'-TYAAGTG-3' and 5'-(A/G) GGTGT-3', respectively [23] [24]. Since these transcription factors also participate in cardiac development [25] and interact with GATA-4 specifically [5] [26], they may facilitate the role of GATA-4 in cardiac differentiation. Although three cis-elements (HF-1-HF-3) located in the MLC-2v promoter were analyzed (Figure 4(b)) [27], the first intronic region further participates in the up-regulation of the MLC-2v gene in cardiac hypertrophy [28]. Such an additional regulatory element may explain the different responses of native and hybrid MLC-2v promoters to DMSO (Figure 3).

Although roles of GATA factors expressed in P19CL6 cells and GATA motifs in the CMV enhancer should be further examined in detail, the specific and in- 
ducible gene expression by means of the CMV enhancer in the cardiac environment may be useful for gene therapy as well as the tracing of differentiated cells [19].

\section{Acknowledgements}

This research was supported in part by a grant from MEXT [Grant-in-Aid for Strategic Medical Science Research Center, 2010-2014 (The MIAST Project)].

\section{Conflict of Interest}

The authors have no conflict of interest.

\section{References}

[1] Habara-Ohkubo, A. (1996) Differentiation of Beating Cardiac Muscle Cells from a Derivative of P19 Embryonal Carcinoma Cells. Cell Structure and Function, 21, 101-110. https://doi.org/10.1247/csf.21.101

[2] Ohara, Y., Atarashi, T., Ishibashi, T., Ohashi-Kobayashi, A. and Maeda, M. (2006) GATA-4 Gene Organization and Analysis of Its Promoter. Biological and Pharmaceutical Bulletin, 29, 410-419. https://doi.org/10.1248/bpb.29.410

[3] Ishibashi, T., Yokura, Y., Ohashi, K., Yamamoto, H. and Maeda, M. (2011) Conserved GC-Boxes, E-Box and GATA Motif Are Essential for GATA-4 Gene Expression in P19CL6 Cells. Biochemical and Biophysical Research Communications, 413, 171-175. https://doi.org/10.1016/j.bbrc.2011.08.036

[4] Ieda, M., Fu, J.-D. Delgado-Olguin, P., VedanTham, V., Hayashi, Y., Bruneau, B.G. and Srivastava, D. (2010) Direct Reprogramming of Fibroblasts into Functional Cardiomyocytes by Defined Factors. Cell, 142, 375-386.

https://doi.org/10.1016/j.cell.2010.07.002

[5] Garg, V., Kathiriya, I.S., Barnes, R., Schluterman, M.K., King, I.N., Butler, C.A., Rothrock, C.R., Eapen, R.S., Hirayama-Yamada, K., Joo, K., Matsuoka, R., Cohen, J. and Srivastava, D. (2003) GATA4 Mutations Cause Human Congenital Heart Defects and Reveal an Interaction with TBX5. Nature, 424, 443-447.

https://doi.org/10.1038/nature01827

[6] Kodo, K., Nishizawa, T., Furutani, M, Arai, S., Ishihara, K., Oda, M., Makino, S., Fukuda, K., Takahashi, T., Matsuoka, R., Nakanishi, T. and Yamagishi, H. (2012) Genetic Analysis of Essential Cardiac Transcription Factors in 256 Patients with Non-Syndromic Congenital Heart Defects. Circulation Journal, 76, 1703-1711. https://doi.org/10.1253/circj.CJ-11-1389

[7] Han, Y., Xu, H., Cheng, J., Zhang, Y., Gao, C., Fan, T., Peng, B., Li, B., Liu, L. and Cheng Z. (2016) Downregulation of Long Non-Coding RNA H19 Promotes P19CL6 Cells Proliferation and Inhibits Apoptosis during Late-Stage Cardiac Differentiation via miR-19b-Modulated Sox6. Cell \& Bioscience, 6, 58. https://doi.org/10.1186/s13578-016-0123-5

[8] Moore, J.C., Spijker, R., Martens, A.C., De Boer, T., Rook, M.B., Van Der Heyden, M.A.G., Tertoolen, L.G. and Mummery, C.L. (2004) A P19Cl6 GFP Reporter Line to Quantify Cardiomyocyte Differentiation of Stem Cells. The International Journal of Developmental Biology, 48, 47-55. https://doi.org/10.1387/ijdb.15005574

[9] Jin, Y., Pasumarthi, K.B.S., Bock, M.E., Chen, Y., Kardami, E. and Cattini, P.A. (1995) Effect of "Enhancer" Sequences on Ventricular Myosin Light Chain-2 Pro- 
moter Activity in Heart Muscle and Nonmuscle Cells. Biochemical and Biophysical Research Communications, 210, 260-266. https://doi.org/10.1006/bbrc.1995.1655

[10] Saiki, R.K., Gelfand, D.H., Stoffel, S., Scharf, S.J., Higuchi, R., Horn, G.T., Mullis, K.B. and Erlich, H.A. (1988) Primer-Directed Enzymatic Amplification of DNA with a Thermostable DNA Polymerase. Science, 239, 487-491. https://doi.org/10.1126/science.2448875

[11] Henderson, S.A., Spencer, M., Sen, A., Kumar, C., Siddiqui, M.A.Q. and Chien, K.R. (1989) Structure, Organization, and Expression of the Rat Cardiac Myosin Light Chain-2 Gene: Identification of a 250-Base Pair Fragment Which Confers Cardiac-Specific Expression. Journal of Biological Chemistry, 264, 18142-18148.

[12] Maeda, M., Asahara, S., Nishi, T., Mushiake, S., Oka, T., Shimada, S., Chiba, T., Tohyama, M. and Futai, M. (1995) The Rat Intrinsic Factor Gene: Its 5'-Upstream Region and Chief Cell-Specific Transcription. The Journal of Biochemistry, 117, 1305-1311. https://doi.org/10.1093/oxfordjournals.jbchem.a124859

[13] Sanger, F., Coulson, A.R., Barrell, B.G., Smith, A.J.H. and Roe, B.A. (1980) Cloning in Single-Stranded Bacteriophage as an Aid to Rapid DNA Sequencing. Journal of Molecular Biology, 143, 161-178. https://doi.org/10.1016/0022-2836(80)90196-5

[14] Sambrook, J., Fritsch, E.F. and Maniatis, T. (1989) Molecular Cloning: A Laboratory Manual. 2nd Edition, Cold Spring Harbor Laboratory, Cold Spring Harbor.

[15] Sato, R., Inoue, J., Kawabe, Y., Kodama, T., Takano, T. and Maeda, M. (1996) Sterol-Dependent Transcriptional Regulation of Sterol Regulatory Element-Binding Protein-2. Journal of Biological Chemistry, 271, 26461-26464. https://doi.org/10.1074/jbc.271.43.26461

[16] Sakai, Y., Nakagawa, R., Sato, R. and Maeda, M. (1998) Selection of DNA Binding sites for Human Transcriptional Regulator GATA-6. Biochemical and Biophysical Research Communications, 250, 682-688. https://doi.org/10.1006/bbrc.1998.9374

[17] Nishi, T., Kubo, K., Hasebe, M., Maeda, M. and Futai, M. (1997) Transcriptional Activation of $\mathrm{H}^{+} / \mathrm{K}^{+}$-ATPase Genes by Gastric GATA Binding Proteins. The Journal of Biochemistry, 121, 922-929. https://doi.org/10.1093/oxfordjournals.jbchem.a021674

[18] Takeda, M., Obayashi, K., Kobayashi, A. and Maeda, M. (2004) A Unique Role of an Amino Terminal 16-Residue Region of Long-Type GATA-6. The Journal of Biochemistry, 135, 639-650. https://doi.org/10.1093/jb/mvh077

[19] Gruh, I., Wunderlich, S., Winkler, M., Schwanke, K., Heinke, J., Blömer, U., Ruhparwar A., Rohde, B., Li, R.-K., Haverich, A. and Martin, U. (2008) Human CMV Immediate-Early Enhancer: A Useful Tool to Enhance Cell-Type-Specific Expression from Lentiviral Vectors. The Journal of Gene Medicine, 10, 21-32. https://doi.org/10.1002/jgm.1122

[20] von Salisch, S., Klar, M., Thurisch, B., Bungert, J. and Dame, C. (2011) Gata4 and Sp1 Regulate Expression of the Erythropoietin Receptor in Cardiomyocytes. Journal of Cellular and Molecular Medicine, 15, 1963-1972. https://doi.org/10.1111/j.1582-4934.2010.01193.x

[21] Schirm, A., Jiricny, J. and Schaffner, W. (1987) The SV40 Enhancer Can Be Dissected into Multiple Segments, Each with a Different Cell Type Specificity. Genes \& Development, 1, 65-74. https://doi.org/10.1101/gad.1.1.65

[22] Cirillo, L.A., Lin, F.R., Cuesta, I., Friedman, D., Jarnik, M. and Zaret, K.S. (2002) Opening of Compacted Chromatin by Early Developmental Transcription Factors HNF3 (FoxA) and GATA-4. Molecular Cell, 9, 279-289. https://doi.org/10.1016/S1097-2765(02)00459-8 
[23] Inga, A., Reamon-Buettner, S.M., Borlak, J. and Resnick, M.A. (2005) Functional Dissection of Sequence-Specific NKX2-5 DNA Binding Domain Mutations Associated with Human Heart Septation Defects Using a Yeast-Based System. Human Molecular Genetics, 14, 1965-1975. https://doi.org/10.1093/hmg/ddi202

[24] Ghosh, T.K., Packham, E.A., Bonser, A.J., Robinson, T.E., Cross, S.J. and Brook, J.D. (2001) Characterization of the TBX5 Binding Site and Analysis of Mutations That Cause Holt-Oram Syndrome. Human Molecular Genetics, 10, 1983-1994. https://doi.org/10.1093/hmg/10.18.1983

[25] Srivastava, D. and Olson, E.N. (2000) A Genetic Blueprint for Cardiac Development. Nature, 407, 221-226. https://doi.org/10.1038/35025190

[26] Durocher, D., Charron, F., Warren, R., Schwartz, R. and Nemer, M. (1997) The Cardiac Transcription Factor Nkx2-5 and GATA-4 Are Mutual Cofactors. The EMBO Journal, 16, 5687-5696. https://doi.org/10.1093/emboj/16.18.5687

[27] Lee, K. J., Hickey, R., Zhu, H. and Chien, K. (1994) Positive Regulatory Elements (HF-1a and HF-1b) and a Novel Negative Regulatory Element (HF-3) Mediate Ventricular Muscle-Specific Expression of Myosin Light-Chain 2-Luciferase Fusion Genes in Transgenic Mice. Molecular and Cellular Biology, 14, 1220-1229. https://doi.org/10.1128/MCB.14.2.1220

[28] Mathew, S., Mascareno, E. and Siddiqui, M.A.Q. (2004) A Ternary Complex of Transcription Factors, Nishéd and NFATc4, and Co-Activator p300 Bound to an Intronic Sequence, Intronic Regulatory Element, Is Pivotal for the Up-Regulation of Myosin Light Chain-2v Gene in Cardiac Hypertrophy. Journal of Biological Chemistry, 279, 41018-41027. https://doi.org/10.1074/jbc.M403578200

[29] Zarraga, A.M., Danishefsky, K., Deshpande, A., Nicholson, D., Mendola, C. and Siddiqui, M.A.Q. (1986) Characterization of 5'-Flanking Region of Heart Myosin Light Chain 2A Gene. Journal of Biological Chemistry, 261, 13852-13860. 


\section{Supplementary}

Figure S1. Nucleotide sequence of pCBVenh/MLC-2 $\mathrm{v}_{\text {pro }} /$ EGFP. Nucleotide sequence of pCBVenh/MLC-2 $\mathrm{v}_{\text {pro }} /$ EGFP (4759 bp) was shown. Orange, blue, green and black letters indicate the sequences for CMV enhancer, MLC-2v promoter, a coding region of GFP and pEGFP-N1, respectively. Restriction enzyme sites for AseI, HindIII, BamHI and NotI were shown in red bold letters (ordered from 5 ' side).

tagttATTAATGCGTTACATAACTTACGGTAAATGGCCCGCCTGGCTGACCGCCCAAC GACCCCCGCCCATTGACGTCAATAATGACGTATGTTCCCATAGTAACGCCAATAGGGA CTTTCCATTGACGTCAATGGGTGGAGTATTTACGGTAAACTGCCCACTTGGCAGTACA TCAAGTGTATCATATGCCAAGTACGCCCCCTATTGACGTCAATGACGGTAAATGGCCC GCCTGGCATTATGCCCAGTACATGACCTTATGGGACTTTCCTACTTGGCAGTACATCT ACGTATTAGTCATCGCTATTACCATGGTGATGCGGTTTTGGCAGTACATCAATGGGCG TGGATAGCGGTTTGACTCACGGGGATTTCCAAGTCTCCACCCCATTGACGTCAATGGG AGTTTGTTTTGAAGCTTGACCCAGAGCACAGAGCATCGTTCCCAGGCCAGGCCCCAGC CACTGTCTCTTTAACCTTGAAGGCATTTTTGGGTCTCACGTGTCCACCCAGGCGGGTG TCGGACTTTGAACGGCTCTTACTTCAGAAGAACGGCATGGGGTGGGGGGGCTTAGGTG GCCTCTGCCTCACCTACAACTGCCAAAAGTGGTCATGGGGTTATTTTTAACCCCAGGG AAGAGGTATTTATTGTTCCACAGCAGGGGCCGGCCAGCAGGCTCCTTGGATCCaccgg tcgccaccatggtgagcaagggcgaggagctgttcaccggggtggtgcccatcctggt cgagctggacggcgacgtaaacggccacaagttcagcgtgtccggcgagggcgaggge gatgccacctacggcaagctgaccctgaagttcatctgcaccaccggcaagctgcccg tgccctggcccaccctcgtgaccaccctgacctacggcgtgcagtgcttcagccgcta ccccgaccacatgaagcagcacgacttcttcaagtccgccatgcccgaaggctacgtc caggagcgcaccatcttcttcaaggacgacggcaactacaagacccgcgccgaggtga agttcgagggcgacaccctggtgaaccgcatcgagctgaagggcatcgacttcaagga ggacggcaacatcctggggcacaagctggagtacaactacaacagccacaacgtctat atcatggccgacaagcagaagaacggcatcaaggtgaacttcaagatccgccacaaca tcgaggacggcagcgtgcagctcgccgaccactaccagcagaacacccccatcggcga cggccccgtgctgctgcccgacaaccactacctgagcacccagtccgccctgagcaaa gaccccaacgagaagcgcgatcacatggtcctgctggagttcgtgaccgccgccggga tcactctcggcatggacgagctgtacaagtaaagcggccgcgactctagatcataatc agccataccacatttgtagaggttttacttgctttaaaaaacctcccacacctccccc tgaacctgaaacataaaatgaatgcaattgttgttgttaacttgtttattgcagctta taatggttacaaataaagcaatagcatcacaaatttcacaaataaagcatttttttca ctgcattctagttgtggtttgtccaaactcatcaatgtatcttaaggcgtaaattgta agcgttaatattttgttaaaattcgcgttaaatttttgttaaatcagctcatttttta accaataggccgaaatcggcaaaatcccttataaatcaaaagaatagaccgagatagg gttgagtgttgttccagtttggaacaagagtccactattaaagaacgtggactccaac gtcaaagggcgaaaaaccgtctatcagggcgatggcccactacgtgaaccatcaccct aatcaagttttttggggtcgaggtgccgtaaagcactaaatcggaaccctaaagggag 
cccccgatttagagcttgacggggaaagccggcgaacgtggcgagaaaggaagggaag aaagcgaaaggagcgggcgctagggcgctggcaagtgtagcggtcacgctgcgcgtaa ccaccacacccgccgcgcttaatgcgccgctacagggcgcgtcaggtggcacttttcg gggaaatgtgcgcggaacccctatttgtttatttttctaaatacattcaaatatgtat ccgctcatgagacaataaccctgataaatgcttcaataatattgaaaaaggaagagtc ctgaggcggaaagaaccagctgtggaatgtgtgtcagttagggtgtggaaagtcccca ggctccccagcaggcagaagtatgcaaagcatgcatctcaattagtcagcaaccaggt gtggaaagtccccaggctccccagcaggcagaagtatgcaaagcatgcatctcaatta gtcagcaaccatagtcccgcccctaactccgcccatcccgcccctaactccgcccagt tccgcccattctccgccccatggctgactaatttttttatttatgcagaggccgagg ccgcctcggcctctgagctattccagaagtagtgaggaggctttttggaggcctagg ctttgcaaagatcgatcaagagacaggatgaggatcgtttcgcatgattgaacaaga tggattgcacgcaggttctccggccgcttgggtggagaggctattcggctatgactgg gcacaacagacaatcggctgctctgatgccgccgtgttccggctgtcagcgcagggge gcccggttcttttgtcaagaccgacctgtccggtgccctgaatgaactgcaagacga ggcagcgcggctatcgtggctggccacgacgggcgttccttgcgcagctgtgctcgac gttgtcactgaagcgggaagggactggctgctattgggcgaagtgccggggcaggatc tcctgtcatctcaccttgctcctgccgagaaagtatccatcatggctgatgcaatgcg gcggctgcatacgcttgatccggctacctgcccattcgaccaccaagcgaaacatcgc atcgagcgagcacgtactcggatggaagccggtcttgtcgatcaggatgatctggacg aagagcatcaggggctcgcgccagccgaactgttcgccaggctcaaggcgagcatgcc cgacggcgaggatctcgtcgtgacccatggcgatgcctgcttgccgaatatcatggtg gaaaatggccgcttttctggattcatcgactgtggccggctgggtgtggcggaccgct atcaggacatagcgttggctacccgtgatattgctgaagagcttggcggcgaatggge tgaccgcttcctcgtgctttacggtatcgccgctcccgattcgcagcgcatcgccttc tatcgccttcttgacgagttcttctgagcgggactctggggttcgaaatgaccgacca agcgacgcccaacctgccatcacgagatttcgattccaccgccgccttctatgaaagg ttgggcttcggaatcgttttccgggacgccggctggatgatcctccagcgcggggatc tcatgctggagttcttcgcccaccctagggggaggctaactgaaacacggaaggagac aataccggaaggaacccgcgctatgacggcaataaaaagacagaataaaacgcacggt gttgggtcgtttgttcataaacgcggggttcggtcccagggctggcactctgtcgata ccccaccgagaccccattggggccaatacgcccgcgtttcttccttttccccacccca ccccccaagttcgggtgaaggcccagggctcgcagccaacgtcggggcggcaggccct gccatagcctcaggttactcatatatactttagattgatttaaaacttcatttttaat ttaaaaggatctaggtgaagatcctttttgataatctcatgaccaaaatcccttaacg tgagttttcgttccactgagcgtcagaccccgtagaaaagatcaaaggatcttcttga gatccttttttctgcgcgtaatctgctgcttgcaaacaaaaaaaccaccgctaccag cggtggtttgtttgccggatcaagagctaccaactcttttccgaaggtaactggctt cagcagagcgcagataccaaatactgtccttctagtgtagccgtagttaggccaccac ttcaagaactctgtagcaccgcctacatacctcgctctgctaatcctgttaccagtgg 
ctgctgccagtggcgataagtcgtgtcttaccgggttggactcaagacgatagttacc ggataaggcgcagcggtcgggctgaacggggggttcgtgcacacagcccagcttggag cgaacgacctacaccgaactgagatacctacagcgtgagctatgagaaagcgccacgc ttcccgaagggagaaaggcggacaggtatccggtaagcggcagggtcggaacaggaga gcgcacgagggagcttccagggggaaacgcctggtatctttatagtcctgtcgggttt cgccacctctgacttgagcgtcgatttttgtgatgctcgtcaggggggcggagcctat ggaaaaacgccagcaacgcggcctttttacggttcctggccttttgctggccttttgc tcacatgttctttcctgcgttatcccctgattctgtggataaccgtattaccgccatg cat// 